\title{
A Bunch of Stapler Pin: An Unusual Nasopharyngeal Foreign Body
}

\author{
${ }^{1}$ Soumyajit Das, ${ }^{2}$ Suvamoy Chakraborty, ${ }^{3}$ Subhasish Mukherjee
}

\section{ABSTRACT}

Objective: To highlight the occurrence of unusual foreign bodies in the nasopharynx in the pediatric age group.

Introduction: Foreign bodies are common in ENT practice universally. At times they may present as emergency requiring urgent intervention and many a times they go unnoticed as these are not suspected. Nasopharyngeal foreign bodies are rare in any age group.

Case report: A 10 months old female child was brought with the history of persistent drooling of saliva and refusal to feed after accidental ingestion of a bunch of stapler pin. Plain radiograph showed a bunch of stapler pin in the nasopharynx with enlarged adenoid tissue and prevertebral soft tissue shadow.

Conclusion: Bunch of stapler pin is an unusual foreign body in the nasopharynx. Care should be taken while removing these foreign bodies. Digital manipulation for removal of such foreign bodies are hazardous and should be avoided at all cost.

Keywords: Foreign body, Nasopharynx, Stapler pin, Unusual site.

How to cite this article: Das S, Chakraborty S, Mukherjee S. A Bunch of Stapler Pin: An Unusual Nasopharyngeal Foreign Body. Clin Rhinol An Int J 2015;8(3):127-129.

Source of support: Nil

Conflict of interest: None

\section{INTRODUCTION}

Foreign bodies are common in day to day ENT practice universally. At times however foreign body in the aero digestive tract may present as a life-threatening situation and requires prompt diagnosis and intervention. Almost any age group may be affected, but the circumstances and the nature of foreign bodies differ among different age groups. Nasopharynx is an uncommon site and foreign bodies here may often be missed if one does not excel a high level of suspicion. We present an interesting case of an impacted foreign body (bunch of stapler pin containing

\footnotetext{
${ }^{1}$ Assistant Professor, ${ }^{2}$ Professor and Head, ${ }^{3}$ Associate Professor

${ }^{1-3}$ Department of ENT, Sikkim Manipal Institute of Medical Sciences, Gangtok, Sikkim, India
}

Corresponding Author: Soumyajit Das, Assistant Professor Department of ENT, Sikkim Manipal Institute of Medical Sciences, Gangtok, Sikkim, India, Phone: 9475713937, e-mail: drsoumya_entamch@rediffmail.com twenty nine pins) in an unusual site nasopharynx in a 10 months old child.

\section{CASE REPORT}

A 10 months old female child was brought by the parents with complaints of persistent drooling of saliva and refusal of feeds for 6 hours. Onset of symptoms following alleged accidental ingestion of a bunch of stapler pins by the child 4 hours ago. An attempt was made at home to remove the bunch of stapler pins by finger manipulation which failed and was followed by irritability of the child and associated symptoms. On examination the child had persistent drooling of saliva, had blood tinged nasal discharge but no frank epistaxis. The child had noisy breathing and was tachypnoeic. There was no obvious signs of respiratory compromise, stridor or cyanosis. All further attempts of clinical examination of the oropharynx was avoided as the child was irritable and to avert the risk of dislodgement of the foreign body and a possible lifethreatening air way emergency. An lateral view $\mathrm{X}$-ray was done for nose, nasopharynx, neck, and anteroposterior view of the chest was done. The X-ray revealed the radiopaque shadow of the bunch of stapler pins lodged in the nasopharynx. The foreign body was impacted on the dorsal aspect of the soft palate. The view was slightly obscured due to the edema over the soft palate and the soft tissue shadow of large adenoids (Fig. 1). The child was prepared and taken up for removal of the foreign body under general anesthesia. An orotracheal tube was passed and the oropharynx packed to arrest slippage of the foreign body downwards. Nasal decongestion was achieved by soft nasal packs soaked in $1 \mathrm{ml}$ of 1:10000 adrenaline. A $30^{\circ}$ pediatric rigid nasal endoscope was negotiated through the left nose and the foreign body was located, impacted on the dorsal aspect of the soft palate and obscured by the mass of the large adenoid tissue. A pediatric feeding catheter was negotiated through the right nasal cavity and delivered in to the oral cavity. The feeding catheter was pulled through the oral cavity to retract the soft palate thereby obtaining a better view of the foreign body. A curved mosquito forceps was negotiated through the oral cavity on to the dorsal aspect of the soft palate, under direct vision of the endoscope the foreign body (Fig. 2) was dislodged and collected in the oral cavity. There was no bleeding 


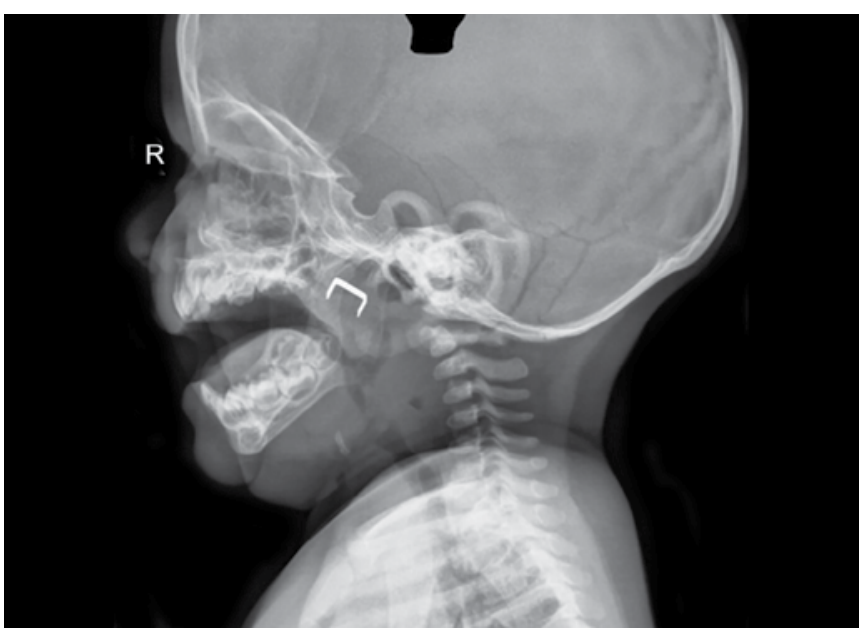

Fig. 1: Skiagram lateral view nasopharynx showing stapler pins in the nasopharynx with an enlarged adenoid tissue. Prevertebral soft tissue shadow is also seen to be increased in the skiagram

and a repeat endoscopy was done to ensure no piece of the bunch of stapler pin was left behind. Nasal soft pack soaked in $0.1 \%$ xylometazoline was retained in the nose and nasopharynx to prevent any overt edema. The packs were removed after 5 minutes. Check X-ray was done postoperatively and it did not reveal any evidence of retained foreign body. The child had an uneventful recovery and was discharged from the hospital after observation for 24 hours.

\section{DISCUSSION}

Foreign bodies in the aerodigestive tract are commonly encountered by an ENT surgeon. These range from coins, batteries, beads, seeds, etc. in children to bones, dentures, etc. in adults. Rare foreign bodies in nasopharynx like thimble ${ }^{1}$ and fish ${ }^{2}$ have also been reported in literature. A bunch of stapler pin containing twenty nine pins is an unusual foreign body in any age group and has not been reported so far in literature. Foreign bodies of the aerodigestive tract mostly involve nose, base of tongue, cricopharynx and larynx, trachea bronchus and esophagus. However nasopharyngeal foreign bodies are rare when compared to other sites and this is due to the wider postnasal space. An ingested foreign body can rarely lodge into the nasopharynx in normal circumstances. However digital manipulation of the foreign body inside the oral cavity may cause it to slip into the nasopharynx as was seen in this case. The foreign body may also lodge in the nasopharynx in case of vomiting and regurgitation or if the foreign body was taken in the mouth in lying down position thereby making the nasopharynx dependent. ${ }^{3}$ The attempt of digital manipulation to try and remove an ingested foreign body can be dangerous as it can cause mucosal injury in the aerodigestive tract leading to soft tissue edema or may dislodge the foreign body down risking airway obstruction in both the situa-

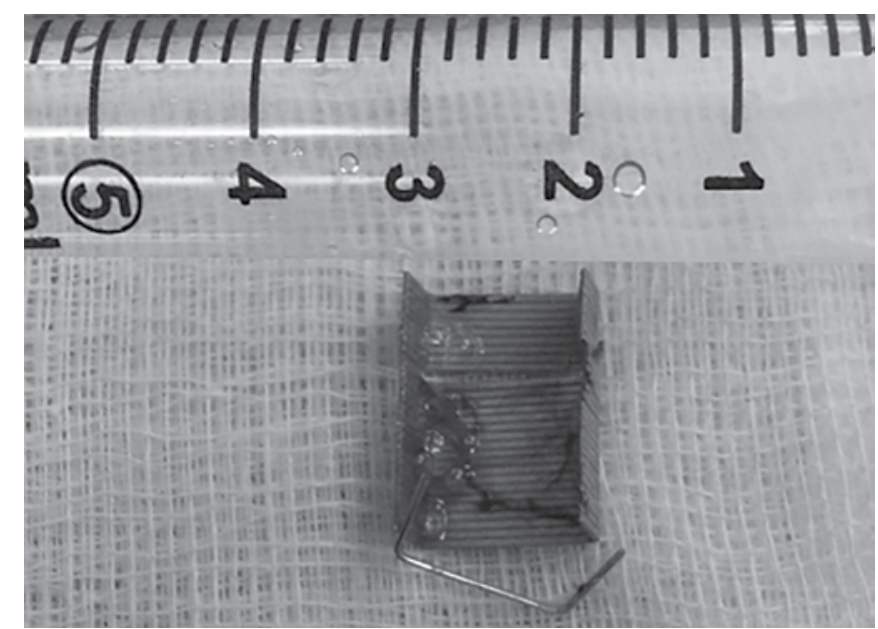

Fig. 2: Bunch of stapler pin removed from the nasopharynx of the patient

tions. Foreign bodies in the nasopharynx may be missed on clinical examination and may accidentally be discovered in a radiograph of the nasopharynx. ${ }^{5}$ Long standing undetected nasopharyngeal foreign bodies have been reported to have caused acquired cleft palate. ${ }^{4}$ Nasopharyngeal foreign bodies can be removed with forceps under endoscopic guidance. However it can pose a challenge in infants because of the narrow nasal passage and hypertrophied adenoid mass as it was in our case. In this particular case it was utmost importance to remove the bunch en masse without dislodging the individual pins. Disintegration of individual pins during removal could have made removal more difficult and traumatic. The presence of adenoid tissue obstructing the choana can make endoscopic visualization difficult particularly when the foreign body remains embedded in the nasophayngeal part of the soft palate as was seen in our case. In this patient the stapler pin could be visualized properly after retracting the soft palate with an infant feeding tube and then using a pediatric rigid nasal endoscope through the opposite nose.

This case is reported because of the unusual nature of the foreign body at an unusual site. We also intend to highlight the circumstances under which the foreign body can get impacted in the nasopharynx in such age group. Last but not the least it is noteworthy to mention the difficulty such foreign bodies in a 10 months old child can pose to the ENT surgeon. Proper instrumentation is required for removal of such foreign bodies taking care that it is removed in a single piece.

\section{SUMMARY}

- Nasopharyngeal foreign bodies are rare.

- Bunch of stapler pin in the nasopharynx has not been reported earlier.

- Attempt to remove such foreign bodies by digital manipulation can be hazardous. 
- Care should be taken while removing these foreign bodies so as not to cause any mucosal injury and airway compromise.

\section{REFERENCES}

1. Kumar J, Sunkum AG. Nasopharyngeal foreign body in an young child. Indian J Otolaryngol Head Neck Surg 2011 JulSept;63(3):285-286.

2. Kumar R, Mudunuri R, Gannavarapu DP, Narasimha A, Mukkamala M, Jameel KM. Unusual foreign body in nose and nasopharynx - a rare case. J Clin Diagnostic Res 2015 Apr;9(4):MD01-MD02.

3. Majumdar PK, Sinha AK, Mookherjee PB, Ganguly SN. An unusual foreign body (10 NP Coin) in nasopharynx. Ind J Otolaryngol Head Neck Surg 1999;52(1):93.

4. Kanotra SP, Kanotra S, Paul J. Cleft palate secondary to an ingested foreign body: a learning experience. J Emerg Med 2012 Nov;43(5):e315-317.

5. Martins RH, Mano JB, da Silva EF. Foreign body in nasopharynx: an accidental radiological finding. Braz J Otorhinolaryngol 2006 May-Jun;72(3):431. 